\title{
Quels savoirs pour l'enseignement du français et de la production écrite au secondaire dans les plans de formation suisses romands?
}

\section{Roxane Gagnon et Marc Surian}

Notre contribution s'inscrit dans un vaste projet de recherche sur les pratiques de formation à l'enseignement de la production écrite. Une analyse en amont des prescriptions institutionnelles des institutions à l'enseignement secondaire est incontournable pour comprendre les pratiques effectives des formateurs. Cet article vise à mettre en évidence, dans un premier temps, les facteurs et les contraintes institutionnels agissant sur les institutions à l'enseignement secondaire en Suisse romande. Dans un deuxième temps, nous situons les unités de formation - cours et séminaires - dédiées à la didactique du français dans l'ensemble des cursus proposés pour la formation des enseignants du secondaires. Enfin, par une analyse fine des contenus des descriptifs des unités de formation nous établissons un essai de catégorisation des contenus de savoirs. Cette dernière permet de dégager cinq ensembles de contenus de savoirs: institutionnels, disciplinaires, liés à l'élève et la classe, liés au travail enseignant et à la pratique. Par ce travail, nous mettons en évidence les similitudes et les divergences entre les différentes institutions de formation à l'enseignement secondaire dans une approche compréhensive. Nous dessinons ainsi les tendances qui permettent de saisir les priorités pour l'enseignement de la production écrite dans les institutions de formation suisses romandes.

\section{Introduction}

Le présent texte fait partie d'un projet plus large sur les pratiques de formation dans le domaine de la production écrite ${ }^{1}$. Nous procédons à une analyse fine des contenus des plans d'études et des descriptifs d'unités de formation à l'enseignement secondaire afin de proposer une catégorisation des contenus de formation en didactique du français et, plus particulièrement, en lien à l'enseignement de la production écrite. L'examen des programmes et des plans d'études des institutions vise à mieux connaitre et mieux comprendre les pratiques des formateurs et le contexte dans lequel ceux-ci interviennent. Nos objectifs sont triples. Il s'agit d'abord de pointer des facteurs contextuels suscep- 
tibles d'influencer les contenus de formation dispensés; l'angle d'approche ici est sociohistorique, car nous présupposons que les pratiques passées teintent les pratiques de formation présentes. En deuxième lieu, nous voulons reconstruire les cursus de formation proposés, de manière à faire ressortir les maquettes des programmes de formation pour l'enseignement du français au secondaire I et II. Les plans de formation regroupent l'ensemble des enseignements que le formé doit/peut recevoir afin de compléter son diplôme en enseignement secondaire dans une institution. En troisième lieu, il s'agit de procéder à un examen minutieux des descriptifs des unités de formation ${ }^{2}$ consacrées à l'enseignement de la production écrite en français de manière à lister les principaux items de contenu et à les catégoriser. Ces trois étapes complétées, nous sommes à même de mieux cerner les pratiques de formation à l'enseignement de la production écrite telles qu'elles se dégagent des documents institutionnels. De plus, cette catégorisation des contenus de savoir de la formation jette les bases à l'analyse des pratiques effectives.

La formation des enseignants regroupe des objets hétérogènes, composites, en interaction les uns avec les autres. Une entrée par les objets propres à la didactique du français permet de circonscrire l'ensemble des savoirs couverts par la discipline "français», et de situer la part des savoirs dédiés à l'enseignement de la production écrite. Néanmoins, nous sommes conscients que cet angle d'analyse ne saurait rendre compte de l'ensemble des cursus des institutions de formation et, notamment, des unités dévolues aux stages pratiques.

\section{Les facteurs contextuels et leurs influences sur la formation dispensée}

L'explicitation des situations sociohistoriques et politiques qui ont façonné et façonnent encore le paysage de la formation à l'enseignement secondaire en Suisse romande est nécessaire à la saisie des objets, des activités et des contenus de savoir. À partir d'une recension d'écrits portant sur l'évolution des institutions de formation suisses romandes au cours du temps, nous mettons en lumière quelques facteurs contextuels susceptibles d'avoir une influence sur les pratiques.

\section{Les premiers pas}

La question d'une formation spécifique aux différents ordres d'enseignement s'est posée dès l'institutionnalisation des formations à l'enseignement au cours du $19^{\mathrm{e}}$ siècle, en lien avec le développement des systèmes éducatifs et la séparation progressive entre enseignement primaire, secondaire et supérieur (Lussi Borer, 2009). C'est au moment de la mise sur pied des écoles normales dans la première moitié du $19^{\mathrm{e}}$ siècle qu'est soulevée la question d'une formation plus adéquate pour les enseignants des écoles secondaires et des gymnases (Criblez, 2004). Ceci sonne le glas de l'ancien système de corporations d'enseignants secondaires à 
l'intérieur des facultés de théologies, de lettres et de sciences. De fait, de nouveaux savoirs professionnels de référence sont proposés: des savoirs pédagogiques théoriques (pédagogie générale, histoire de la pédagogie, didactique générale) viennent s'adjoindre aux savoirs disciplinaires existants (Lussi Borer, 2009).

En 1865, quand la direction de l'école polytechnique de Zurich formule le souhait d'instaurer une formation à l'enseignement secondaire, les cantons réagissent par «la mise en place d'offres régionales ou le plus souvent cantonales» (Criblez, 2004). On assiste alors à l'instauration de deux modèles de formation. Le premier distingue le secondaire I du secondaire II, le second unit les deux filières. La séparation de deux ordres du secondaire vise à répondre adéquatement aux configurations économiques et sociales de l'époque: le secondaire inférieur répond au besoin croissant d'une main-d'œuvre qualifiée; le supérieur, quant à lui, a pour but de former la future élite. La séparation secondaire I et II devient dès lors un élément de différenciation entre les cantons alémaniques (qui distinguent) et les cantons romands (qui unissent) ${ }^{3}$.

\section{Aperçu de l'évolution de la formation à l'enseignement secondaire par canton} À Genève, dès le début du siècle jusqu'en 1965, l'université délivre des certificats pédagogiques qui figurent comme des compléments aux licences données. À partir de 1965, et ce, jusqu'en 2008, la "formation des enseignants est complètement désuniversitarisée et connait successivement deux instituts qui dépendent des directions de l'enseignement secondaire cantonal [...], les Études pédagogiques de l'enseignement secondaire (EPES), relayées par l'Institut de formation des maitres de l'enseignement secondaire (IFMES)»(Extermann, 2007, p. 109). En effet, l'augmentation démographique et l'émergence de nouveaux établissements secondaires conduisent à une pénurie d'enseignants, ce qui oblige les autorités cantonales à proposer une institution de formation spécifique. C'est à partir de 2008 que la formation des enseignants pour le secondaire est de nouveau rattachée à l'université sous la forme d'un Institut universitaire pour la formation des enseignants (IUFE).

C'est dans les années 1940 que le canton de Neuchâtel met sur pied un programme de formation. Celui-ci comporte deux volets distincts: le premier, en lien avec les savoirs disciplinaires et l'enseignement théorique en pédagogie, est sous la responsabilité de l'université, tandis que le second, qui concerne la formation à la pratique, est confiée au Département de l'instruction publique. Dès 1985, on assiste à la création d'un Séminaire pédagogique de l'enseignement secondaire, entité autonome de l'université. Dès 2001, la création de la HEP Bejune, qui réunit la formation des enseignants des cantons de Berne, du Jura francophone et de Neuchâtel prend à charge la formation à l'enseignement secondaire. Cette fusion a été opérée pour obtenir une masse critique suffisante selon les exigences de la CDIP4

Dans le canton de Vaud, se crée le Séminaire pédagogique de l'enseignement secondaire (SPES). Cette offre de formation s'inscrit dans le sillage 
du Règlement pour la formation pédagogique des maitres secondaires de 1959. La formation dispensée au SPES, d'une durée d'un an, est organisée pour accueillir les nouveaux licenciés de l'université désirant s'orienter vers l'enseignement. Lors de sa constitution, le SPES rencontre une forte résistance de la part des professeurs et dirigeants de l'Université de Lausanne, non convaincus du bienfondé et de l'adéquation de la formation proposée (Noverraz, 2008). La légitimité du SPES se voit progressivement assurée au tournant des années 1990. Il fermera ses portes en 2002, une année suivant la reprise des activités de formation par la Haute École Pédagogique (HEP).

Avant 1997, il n'y a pas de tradition de formation secondaire dans le canton du Valais. Les licenciés universitaires peuvent accéder au statut d'enseignant sans une véritable formation professionnelle. Par ailleurs, en raison d'un paysage scolaire particulier - des différences importantes existent entre les établissements selon la démographie et la géographie de certaines régions valaisannes - les besoins en personnel qualifié sont hétérogènes. À partir de 1994, une formation pour l'enseignement est exigée (Gaillard, 2004). Dès lors pour satisfaire cette obligation le canton du Valais entretient un partenariat étroit avec l'université de Fribourg. Le canton se tourne aussi vers le SPES, dans le canton de Vaud, en raison d'une demande croissante de formation. Les réquisitions de la CDIP en vue d'une harmonisation des études et l'ouverture de la HEP Valais en 2001 vont permettre au canton du Valais de subvenir de manière autonome aux besoins de formation des futurs enseignants du secondaire valaisan.

Le canton de Fribourg, quant à lui, à l'image de ce qui se produit en Suisse alémanique en matière de formation à l'enseignement secondaire, conserve l'ensemble de la formation professionnelle à l'Université (Lussi Borer, 2009). C'est l'Institut de pédagogie qui en prend la responsabilité, dès 1949. L'administration scolaire certifie la formation qui y est dispensée à travers un diplôme d'État. Malgré l'ouverture de la HEP Fribourg en 2002, qui prend la charge de la formation des enseignants pour le primaire, son équivalent pour le secondaire est maintenu au sein de l'Université.

\section{Et maintenant? Vers une harmonisation romande}

En 2006, à la suite d'un arrêté fédéral, une révision constitutionnelle contraint les Cantons et la Confédération à collaborer dans le domaine de la formation; celle-ci est d'ailleurs désormais soumise à des règles uniformes pour l'ensemble du territoire suisse. Cette révision constitutionnelle poursuit quatre objectifs fondamentaux: favoriser la mobilité, maintenir la souveraineté cantonale, coordonner l'enseignement de niveau universitaire, renforcer la formation professionnelle. Dans le but de créer un Espace romand de la Formation, la CIIP5 a proposé un plan d'études commun à la Suisse romande (Plan d'études romand, désormais PER) et toute une série de mesures de coordination et d'harmonisation: fixation du début de l'école obligatoire à quatre ans, harmonisation progressive de la structure scolaire de l'école obligatoire. Cette série d'initiatives sous-tend une 
volonté politique d'uniformiser progressivement le «processus» et le «produit» de la formation en Suisse romande.

Cette esquisse historique de l'évolution des institutions révèle des disparités quant à la cartographie des savoirs dans les institutions suisses romandes: place des savoirs pédagogiques théoriques (pédagogie générale, histoire de la pédagogie, didactique générale), place des savoirs disciplinaires, place des savoirs professionnels et de la pratique. La jeunesse relative de certaines formations (la HEP Valais, par exemple) ou la prise en charge relativement nouvelle des savoirs dits pratiques peuvent générer des rapports différents entre l'institution de formation et le milieu professionnel. La persistance des traces d'une distinction de mission entre les cursus de formation à l'enseignement du secondaire I et ceux en vue du secondaire II peut entrainer des distinctions quant aux finalités et aux savoirs de la formation des enseignants de ces filières.

Ce bref détour historique sert à mieux saisir les orientations actuelles dans la formation et les diverses transformations passées qui sont susceptibles de se répercuter sur les pratiques des formateurs (Chervel, 1998). Restreignons maintenant notre focale et intéressons-nous plus précisément à la formation à l'enseignement $\mathrm{du}$ français dans les institutions examinées.

\section{Place de La didactique du français et de L'enseignement de la production écrite dans les institutions de formation du secondaire}

Afin de situer les objets Didactique du français et, en son sein, Enseignement de la production écrite, nous avons recueilli une série de documents durant l'année académique 2009-2010: les programmes de formation, les chronogrammes et descriptifs des unités de formation. Nous avons examiné les plans de formation en vue d'établir la liste de toutes les unités obligatoires et facultatives ayant trait à la didactique du français et, plus précisément, à la production écrite. Les programmes de formation ont été obtenus par l'intermédiaire des sites Internet des différentes institutions ou par la prise de contact avec les responsables des programmes ou les formateurs.

Dans une première lecture des plans de formation de chacune des institutions, nous nous sommes intéressés à la durée globale de la formation et aux différentes filières d'enseignement. Nous avons ensuite repéré toutes les unités de formation consacrées à la didactique du français. Le pointage de ces unités consacrées à la didactique du français permet d'obtenir un portrait précis du cursus en français, de voir la part du domaine et les grandes thématiques traitées. Ainsi, pour chaque canton, nous obtenons les différentes variantes de cursus de formation à l'enseignement secondaire en français.

Nous détaillons les différentes formes possibles de la formation à l'enseignement secondaire dans les institutions, en considérant les types de filières, le 
nombre d'années de formation et les intitulés des unités de formation proposées. Nous précisons le nombre de crédits alloués à la didactique du français dans les cursus.

\section{L' IUFE}

L'Institut Universitaire de Formation des Enseignants à Genève (IUFE) ne fait pas de distinction entre les filières du secondaire 1 et du secondaire 2. Tous les étudiants qui envisagent enseigner aux deux cycles suivent les mêmes cours, durant les deux années de formation.

Pour s'engager dans cette formation intitulée Master en enseignement secondaire I et II (MASE), le candidat doit être titulaire d'un master en lettres et avoir la possibilité d'effectuer des heures d'enseignement dans un établissement du canton. Cependant l'IUFE permet l'accès aux enseignants ayant des parcours académiques et professionnels différents. Dans le cas où les étudiants n'auraient pas obtenu leur diplôme de master ou des heures d'enseignement, ils peuvent s'inscrire au Certificat complémentaire de base en didactique de la discipline et en sciences de l'éducation (CCDIDA). Une fois que le titre ou les heures d'enseignement requis sont obtenus, ils peuvent alors poursuivre le cursus MASE en deuxième année. Les étudiants formés à l'enseignement d'une autre discipline que le français, avec toutefois des compléments pour cette branche, suivent le Certificat de spécialisation de formation approfondie en didactique d'une deuxième discipline d'enseignement (CSD2) d'une durée d'un an.

Les trois programmes de l'IUFE sont composés des mêmes unités de formation. En première année, les étudiants suivent le cours Discipline de référence et discipline scolaire et l'atelier Transposition didactique et analyse des pratiques didactiques propres à la discipline dans deux niveaux d'enseignement. Pour la deuxième année du Master, deux ateliers sont proposés: Planification didactique et gestion des apprentissages dans les deux niveaux d'enseignement, ainsi qu'Usage et approche des MITIC. Le nombre de crédits alloués en didactique du français en première année est de 12 sur 30, et de 9 sur 64 pour l'année suivante. Ce qui donne un total de 21 crédits sur 94 en fin de cursus $(23,3 \%)$.

\section{La HEP BEJUNE}

La HEP BEJUNE propose trois formations distinctes pour l'enseignement secondaire: une formation pour le secondaire I, une formation combinée pour le secondaire I et II et une formation pour le secondaire II. Le nombre d'étudiants et la disponibilité des formateurs en didactique du français font en sorte que ces filières sont regroupées. Aussi, les étudiants inscrits dans la Filière A: secondaire I et ceux de la Filière B: secondaire I et écoles de maturité (Sec II) suivent les mêmes cours durant les deux années de formation. La première année, ce sont les séminaires Didactique du français: séminaires spécifiques; Didactique du français: séminaires communs ainsi que deux unités portant sur les technologies de la communication: TICE, cours à option et TP de TICE qui sont proposés. 
En deuxième année, ces unités de formation sont reconduites, elles portent d'ailleurs les mêmes intitulés. Quant au nombre de crédits alloués à la didactique du français, il est de 7 sur 30 en première année et 7 sur 60 en deuxième. Nous obtenons ainsi un total de 14 sur 90 à la fin du parcours (15,6\%).

\section{La HEP Vaud}

À la HEP Vaud, une distinction est faite entre la formation proposée pour les étudiants désireux d'enseigner au secondaire I et ceux convoitant le secondaire II. Quatre facteurs distinguent les deux cursus: a) la nature du diplôme de fin d'études: Master en enseignement pour secondaire I et Diplôme d'enseignement pour le degré secondaire II; b) les prérequis à l'entrée: les étudiants inscrits dans la filière du secondaire II doivent être titulaires d'un master dans une discipline; c) la durée de la formation: deux ans pour la formation au secondaire I, une année pour celle du secondaire II; d) les contenus proposés dans les unités des deux filières, malgré leurs mêmes intitulés. Les deux cursus (secondaire I et II) ont en commun cinq unités:

- deux cours, Savoirs fondamentaux en didactique du français, Didactique du français et éducation aux médias;

- trois séminaires liant la didactique du français et l'éducation aux médias, Intégrer les médias à l'argumentation, Intégrer les médias à l'information, Intégrer les médias à la narration. Ces trois séminaires sont associés au cours d'éducation aux médias.

D'autres unités composent ces deux filières: Pratiques d'enseignement et savoirs fondamentaux; Pratique réflexive en didactique du français, Approfondissements en didactique du français. Bien qu'elles soient chapeautées par le même intitulé, ces unités se distinguent par le formateur responsable et par les contenus inscrits dans le descriptif.

Le nombre de crédits est de 12/120 pour le secondaire I et 12 sur 60 pour le secondaire II (soit respectivement $10 \%$ et $20 \%$ ).

\section{La HEP Valais}

Trois filières de formation sont offertes à la HEP Valais: un Master enseignement secondaire I, un Diplôme d'enseignement secondaire II ainsi qu' un Diplôme combiné d'enseignement secondaire I et II. La formation pour enseigner au secondaire I dure trois ans et celle pour le secondaire II se réalise sur deux années. En ce qui concerne la formation combinée secondaire I et II, elle est programmée sur trois ans. Pour toutes les filières, les unités de formation traitant de la didactique du français sont prévues au cours des deux premières années. Pour le secondaire I et le secondaire II, les unités de formation suivantes sont offertes en première année: Didactique 1- Français L1 et Analyse de pratiques du point de vue didactique français L1; la deuxième année comprend les cours Didactique 2-français L1 et Atelier Didac-TIC français L1. Bien que les intitulés soient les mêmes pour les deux filières, chacune de ces unités se distingue par son contenu 
et son formateur responsable. Les crédits alloués pour le secondaire I totalisent 10 crédits sur $110(9 \%)$. Pour le secondaire II, nous obtenons un total de 10 crédits sur $60(16.7 \%)$.

La filière diplôme d'enseignement secondaire I et II s'organise de manière particulière. Les étudiants choisissent leur programme selon le parcours voulu et, surtout, en fonction des postes occupés en cours de formation. En première année, ils suivent les unités de formation prévues pour la filière du secondaire I, s'ils occupent un poste dans le secondaire I ou s'ils sont sans emploi. Ils peuvent également commencer par les unités du secondaire II dans le cas où ils ont une place dans cet ordre d'enseignement. En deuxième année, les étudiants doivent compléter leur formation en suivant les unités de la seconde filière ainsi que deux autres unités, Analyse des pratiques du point de vue didactique et Didactique complémentaire degré secondaire 2-Français L1. Les unités en lien à la didactique du français valent 15 crédits sur les 110 crédits du cursus global (13.6\%).

\section{L'Université de Fribourg}

L'Université de Fribourg propose deux filières menant à l'obtention de deux diplômes distincts: le Diplôme d'aptitude à l'enseignement secondaire I (DAES 1) et le Diplôme d'aptitude à l'enseignement secondaire II (DAES 2).

Pour le DAES 1, deux variantes sont possibles. La première, Variante 1, constitue la voie classique et prépare à l'enseignement de trois à quatre branches au secondaire I. Cette variante requiert l'obtention d'un Bachelor spécifique de 180 crédits et d'un master valant 90 crédits. Elle exige donc l'obtention d'un total de 270 crédits. La formation scientifique a lieu au sein des facultés; la formation pédagogique est du ressort du Centre d'enseignement et de recherche pour la formation des enseignants.

La Variante 2 prépare à l'enseignement de deux branches et implique l'obtention d'un Bachelor et d'un Master. Le Bachelor de 180 crédits se fait au sein d'une faculté. La formation pédagogique et didactique se réalise dans le cadre du Master de 120 crédits.

L'unité de formation dispensée respectivement en première et en deuxième année de la Variante 1 s'intitule Didactique du français langue maternelle. Elle vaut 10 crédits en première année et 2 en deuxième année, ce qui représente un total de 12 crédits ( $12 / 120$ donc $10 \%)$.

Les étudiants de la variante 2 suivent le même cours donné à la variante 1 en deuxième année - Didactique du français langue maternelle - et obtiennent 12 crédits sur $120(10 \%)$

La formation en vue de l'obtention du DAES 2 se déroule sur une année uniquement. L'unité Didactique du français langue maternelle, bien qu'elle porte le même intitulé, est indépendante des deux autres, elle compte pour 9 crédits sur un total de $60(15 \%)$.

De manière à aider notre lecteur à mieux se représenter ces diverses maquettes de formation à l'enseignement du français au secondaire, nous avons schématisé les cursus de formation dans le tableau 1. 
La reconstitution de ces différentes maquettes relatives à la formation à l'enseignement du français au secondaire donne à voir des cursus variés. Les itinéraires se distinguent dans la durée (1-2-3 ans), les variantes offertes, tant du point de vue de l'accès à la formation (maturité, bachelor, master, emploi ) que des filières offertes (secondaire I, secondaire II, secondaire I et II, filière unique). L'IUFE est la seule institution à ne pas scinder la formation pour le secondaire I et le secondaire II. À BEJUNE, les filières sont scindées, mais l'offre de formation est identique. Est-ce dû à des effectifs plus limités, voire à une volonté d'uniformiser la formation? Le canton de Fribourg prolonge la tradition alémanique qui relègue la formation primaire à une Haute école et le secondaire à l'Université.

Les intitulés des diverses unités de formation reflètent peu les savoirs de la discipline scolaire «français», mis à part l'unité «Savoirs fondamentaux en didactique du français» et celles en lien à l'intégration de la narration, de l'information et de l'argumentation dans les TICS à la HEP Vaud. Les intitulés font apparaitre des aspects liés à la formation professionnelle, «analyse de pratiques», "pratique réflexive», "planification», "gestion des apprentissages». Les intitulés génériques ne permettent pas d'émettre des suppositions au regard du traitement de l'enseignement de la production écrite, encore moins d'une différenciation des contenus selon que les étudiants se destinent au cycle un ou deux du secondaire. Le terme didactique, employé comme adjectif ou substantif, y est omniprésent. Notons que, dans les cantons bilingues (Valais et Fribourg), le terme français est complété par ceux de langue première et de langue maternelle.

La part des unités consacrées à la didactique du français varie substantiellement, les pourcentages se situant entre $10 \%$ et $25 \%$ selon les institutions.

\section{Répartition des contenus des descriptifs de formation}

Pour bâtir une grille d'analyse des contenus de savoir des unités de didactique $\mathrm{du}$ français, nous nous sommes d'abord appuyés sur une recension d'écrits présentant diverses catégorisations des savoirs de la formation. Cinq essais de catégorisation ont servi de base à l'élaboration de notre grille d'analyse; nous avons repris les taxonomies de Chevallard (1999), Lenoir \& Vanhulle (2005), Goigoux, Ria et Toczek-Capelle (2009), Desjardins et Dezutter (2009) ainsi que d'Hofstetter et Schneuwly (2009). Nous avons procédé à leur comparaison, tentant de voir les catégories les plus adaptées aux items de contenu dégagés des descriptifs. Nous avons classé les items de contenu des descriptifs des unités de formation dans un tableau regroupant l'ensemble des cinq propositions des didacticiens. Procédant dans un mouvement d'allers-retours entre théorisation des catégories et classement des items de contenu des descriptifs, notre objectif était d'obtenir la catégorisation la plus étanche possible, de circonscrire des zones de catégories franches. Cette délimitation devait également être en accord 


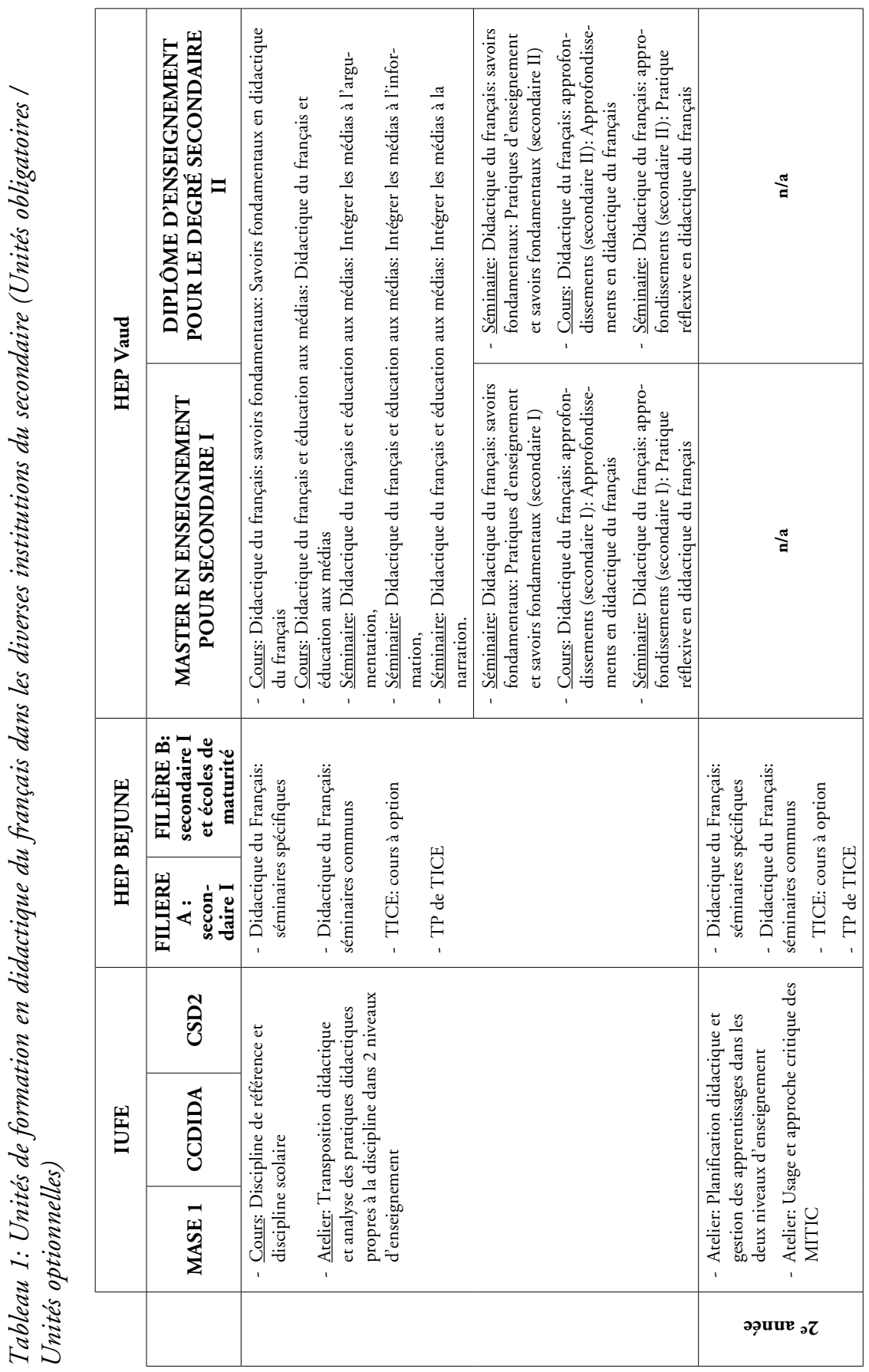




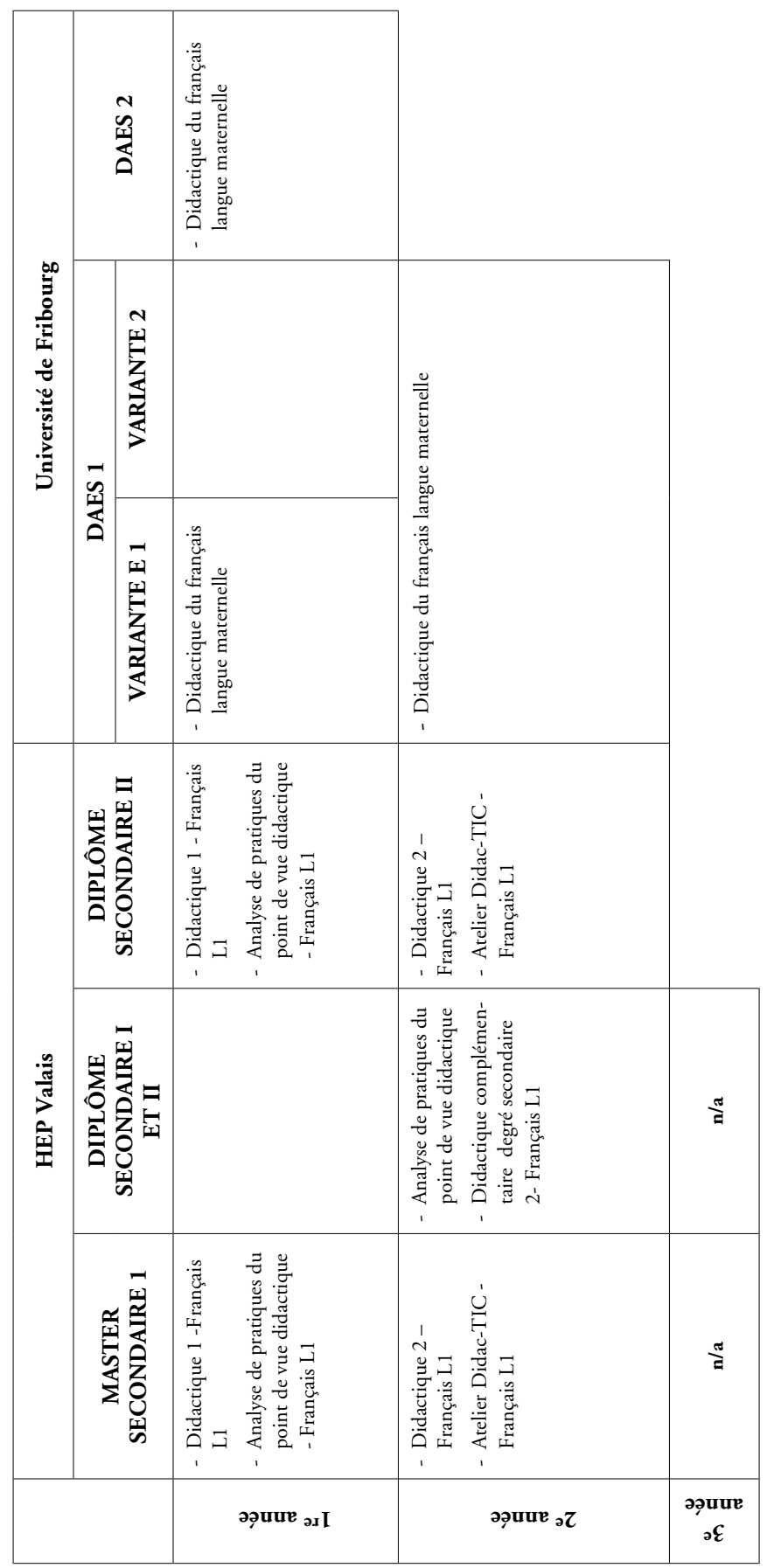


avec notre vision théorique préalable de l'activité enseignante, laquelle prend la forme de gestes didactiques, se déploie à l'aide d'outils et d'un dispositif (Dolz, Schneuwly \& Thévenaz, 2008; Schneuwly \& Dolz, 2009).

Cinq grandes catégories ont ainsi émergé: l'institution, la discipline, l'élève et la classe, l'enseignant et les stages pratiques. Nous avons dû en ajouter une sixième, relative aux modalités de présentation ou aux consignes d'évaluation des différentes unités de formation ${ }^{6}$. Pour chacune des grandes catégories, nous avons dégagé différentes sous-catégories, lesquelles ont été déterminées à postériori en fonction des différents items de contenu examinés.

\section{Corpus analysé}

Les documents examinés ne sont pas dénommés de la même manière selon les institutions et épousent des structures différentes. Les intitulés de diverses rubriques de contenus varient: certaines utilisent les termes objectifs et compétences, d'autres utilisent les termes de thèmes, d'activités, de descriptifs ou de contenus. Le tableau 2 présente les intitulés des documents consultés, les différentes rubriques examinées ainsi que des exemples d'items de contenu pour chacune des institutions.

Tableau 2: Types de documents examinés et intitulés des rubriques des descriptifs consultés pour chaque institution

\begin{tabular}{|c|c|c|c|c|c|}
\hline $\begin{array}{l}\text { Insti- } \\
\text { tution }\end{array}$ & HEP BEJUNE & $\begin{array}{c}\text { Uni } \\
\text { FRIBOURG }\end{array}$ & HEP VAUD & $\begin{array}{c}\text { HEP } \\
\text { VALAIS }\end{array}$ & $\begin{array}{c}\text { IUFE } \\
\text { (UNIGE) }\end{array}$ \\
\hline $\begin{array}{l}\text { Intitulé } \\
\text { des docu- } \\
\text { ments }\end{array}$ & Plan de cours & $\begin{array}{l}\text { Programmes } \\
\text { DAES } 1 \\
\text { et } 2\end{array}$ & $\begin{array}{l}\text { Livret de } \\
\text { cours }\end{array}$ & $\begin{array}{l}\text { Descriptif } \\
\text { de l'Unité } \\
\text { d'Ensei- } \\
\text { gnement }\end{array}$ & $\begin{array}{l}\text { Calendrier } \\
\text { des ateliers, } \\
\text { calendrier des } \\
\text { séminaires }\end{array}$ \\
\hline Rubriques & $\begin{array}{l}\text { - Objectif } \\
\text { général du } \\
\text { cours } \\
\text { - Objectifs spéci- } \\
\text { fiques du cours } \\
\text { - Compétences } \\
\text { professionnelles } \\
\text { visées } \\
\text { - Description et } \\
\text { organisation du } \\
\text { cours }\end{array}$ & $\begin{array}{l}\text { - Modules de } \\
\text { cours } \\
\text { - Dates } \\
\text { proposées } \\
\text { - Contenus } \\
\text { - Savoir-faire }\end{array}$ & $\begin{array}{l}\text { - Connaissances } \\
\text { préalables } \\
\text { recommandées } \\
\text { - Compétences } \\
\text { travaillées et } \\
\text { niveaux de } \\
\text { maitrise évalués } \\
\text { - Thèmes et } \\
\text { activités clés } \\
\text { - Ouvrages de } \\
\text { base }\end{array}$ & $\begin{array}{l}\text { - Compétences } \\
\text { travaillées } \\
\text { - Objectifs } \\
\text { - Thèmes, } \\
\text { concepts, } \\
\text { notions }\end{array}$ & $\begin{array}{l}\text { - Dates } \\
\text { - Thèmes }\end{array}$ \\
\hline $\begin{array}{l}\text { Exemple } \\
\text { d'item de } \\
\text { contenu }\end{array}$ & $\begin{array}{l}\text { Enseignement } \\
\text { de l'écrit et } \\
\text { évaluation }\end{array}$ & $\begin{array}{l}\text { Expression écrite } \\
\text { et orale à partir } \\
\text { des plans de } \\
\text { cheminement }\end{array}$ & $\begin{array}{l}\text { Écriture: diversité } \\
\text { des situations, } \\
\text { exploration des } \\
\text { démarches, analyse } \\
\text { de textes d'élèves }\end{array}$ & $\begin{array}{l}\text { Genres de textes et } \\
\text { types de discours }\end{array}$ & $\begin{array}{l}\text { Enseignement de } \\
\text { l'orthographe; } \\
\text { présentation de la } \\
\text { grille simplifiée } \\
\text { élève }\end{array}$ \\
\hline
\end{tabular}

À l'intérieur des différentes rubriques, les items de contenu des unités sont formulés par le biais d'un verbe à l'infinitif suivi d'un complément (HEP BEJUNE, Valais, HEP Vaud) ou prennent la forme de syntagmes nominaux 
(HEP BEJUNE, HEP Vaud, HEP Valais, IUFE, Uni Fribourg). Ces aspects formels nous ont ainsi permis de délimiter les items. Ceux-ci ont ensuite été extraits des rubriques.

\section{Répartition des items de contenu en catégories de savoir}

L'élaboration d'une grille d'analyse des contenus de savoir, dans les descriptifs de formation à l'enseignement de la production écrite, vise à identifier les objets de la formation. Nous les détaillons dans le tableau ci-dessous. Les chiffres proposés dans la colonne Total correspondent au nombre d'items de contenus issus des différents descriptifs des institutions de formation romandes. Le classement des items dans notre grille d'analyse nous a permis de détailler les cinq grandes catégories en sous-catégories.

Les contenus de savoir sur les prescriptions institutionnelles rassemblent seulement 11 items sur les 253 items totaux, soit 4,3\%. Ils sont répartis en trois sous-catégories: les finalités, les plans d'études et les moyens d'enseignement officiels.

Les finalités regroupent deux items, L'activité des élèves, objectifs d'apprentissage et finalités ainsi que Lecture/littérature: objectifs. Huit items portent sur les plans d'études; ceux-ci concernent la présentation du PER, Présentation et évaluation place de la production de textes, PER ou les plans d'études des cantons: Enseignement du français au CO et au collège: plans de cheminement et instructions officielles. Des items concernant l'analyse et la progression des plans d'études sont également suggérés: Analyser des compétences décrites dans le plan d'études et Plans d'étude, programmes: quelle progression? Au sujet des moyens d'enseignements officiels, nous n'avons relevé qu'un seul item, Enseignement de la grammaire: manuels de référence (Nathan-Hatier).

Les contenus de savoir sur le français et ses disciplines représentent la deuxième catégorie en importance, avec $30 \%$ du total des items (76/253).

Ils se divisent en deux sous-catégories: les contenus de savoir sur la discipline français (39/76) et les contenus de savoir sur la didactique du français (37/76). La première comprend la Structuration de la langue, qui occupe une part majoritaire des items de contenu (14/76). Cela englobe des items liés à l'orthographe, L'orthographe rénovée ou Plurisystème orthographique, et des items relatifs à la grammaire. Là encore, nous pouvons distinguer des items traitant de la Grammaire de la phrase, d'autres, plus nombreux, en lien à la Grammaire du texte. Nous retrouvons également des items plus génériques tels que Grammaire: terminologie et sens, et d'autres plus liés au cursus des élèves Grammaire spécifique au collège. S'ajoutent également dans les contenus de savoir sur la discipline français les théories du langage et des genres textuels, qui englobent 9 items sur 76. Ces 9 items portent sur l'étude des genres textuels, Définition d'un genre, ou Études "genres» en littérature ou l'analyse du discours, par exemple Modèle 
psychologique des discours. Cette sous-catégorie couvre aussi les items pour la Production orale (3/76), comme Production de textes oraux. Les items Le résumé, la synthèse et Les genres épistolaires ont été classés dans Production écrite (2/76). Viennent ensuite dans cette sous-catégorie les Références culturelles (11/76). Celles-ci regroupent les contenus abordant la littérature ou les auteurs littéraires: Ouverture sur la littérature suisse-romande et les littératures francophones: Possibilités de lecture et panorama d'cuvres, Lecture d'une ouvre intégrale: le roman ou la nouvelle, Présences ordinaires de la littérature (noms de rue, cinéma, jeux vidéos, figurines) ou, encore, Rousseau et Genève: $300^{\circ}$ anniversaire de sa naissance (visite guidée). Nous y avons également classé un item portant sur l'extrait littéraire, Regard critique sur les différentes approches de l'extrait littéraire. Y sont inclus également les items ayant trait à la poésie, au théâtre et au cinéma. En voici quelques exemples: Rappel: figures de style - la versification, Rappel: le dialogue au théatre: l'argumentation ou encore Mise en pratique: texte et film; texte et image. Enfin, cette sous-catégorie comprend le Français et le monde du travail (1/76), qui contient un seul item la «communication professionnelle» au secondaire 2.

La deuxième sous-catégorie les contenus de savoir sur la didactique du français (37/76) comporte les éléments liés à l'histoire de l'enseignement (8/76). Nous retrouvons donc des items traitant soit de l'histoire de l'enseignement du français, d'une de ces sous-disciplines, d'un genre d'activité scolaire: Situer historiquement différents courants de l'enseignement du français; Histoire de l'enseignement grammatical; Histoire de l'enseignement de la production écrite; Histoire de l'enseignement de la littérature; Enseignement de la dissertation: éléments historiques ainsi qu'Explication de textes du $16^{e}$ au $20^{e}$ siècle: la lecture méthodique. Dans cette sous-catégorie, nous avons également classé les contenus de savoir et les théories sur les conceptions de l'enseignement (8/76). Ils comprennent les items liés aux phénomènes de transposition: Transposition didactique; dédoublement générique impliqué par la scolarisation d'un genre ou encore outils de langue: savoirs savants et savoirs à enseigner. Certains renvoient au Cadre dans lequel se déroule l'enseignement du français, d'autres à des aspects plus généraux liés à la séquence didactique: La séquence - la leçon: théories. Enfin viennent tous les items de contenu qui sont liés à une didactique d'une sous-discipline du français: Didactique de l'orthographe (2/76), Didactique du lexique (3/76), Didactique de l'expression écrite (6/76), Didactique de l'expression orale (3/76), Didactique de la lecture et de la littérature (4/76), Didactique de la poésie (4/76) et Didactique de l'argumentation (1/76).

Les contenus de savoir sur les élèves et leurs apprentissages restent faiblement représentés et occupent une proportion similaire à celle des contenus de savoir portant sur les prescriptions institutionnelles (13 items sur 253, soit $5.1 \%$ ). La plupart des occurrences répertoriées sont associées à la sous-catégorie Analyse des textes d'élèves (8 items sur 13 de cette catégorie). Les items sont d'abord 
génériques - Travail sur des copies d'élèves; Écriture: analyse des textes d'élèves. D'autres sont davantage ciblés sur le rôle de ces analyses dans le déploiement des dispositifs d'apprentissage, comme Analyse de copies d'élèves comparaison PI et PF en argumentation; Analyse des productions et effet sur l'enseignement modulaire. Le restant des items est attaché à l'évaluation: Évaluation et correction: les travaux écrits et oraux et leur évaluation; Typologie et hiérarchisation des erreurs orthographiques.

La sous-catégorie contenus de savoir sur les processus d'apprentissage rassemble deux items uniquement: Détecter forces et difficultés des élèves; Lecturellittérature: processus de compréhension. Enfin les items relatifs aux contenus de savoir sur sa propre pratique de scripteur concernent les ateliers d'écriture - Production d'un genre ou Atelier d'écriture: produire des textes variés - et la mise à distance de ces ateliers-Retour sur l'atelier d'écriture: analyser les productions à l'aide des outils fournis au cours.

Les contenus de savoir sur les situations et les gestes didactiques occupent la part la plus importante $(53 \%)$ des contenus, avec 134 items sur 253 . La première sous-catégorie concerne le geste Planifier la matière. Seulement deux items de contenu y font référence: Questions de planification; Planifier des séquences d'enseignement/ apprentissage dans la branche.

La sous-catégorie Mettre en place des dispositifs d'enseignement contient plus de la moitié des items de cette catégorie (83/134). Elle se décline en deux groupes. Le premier englobe les items autour de l'analyse des dispositifs didactiques. Certains ont trait aux séquences d'enseignement en général: Les séquences didactiques (SD) modèles d'hier et d'aujourd'hui; La séquence - la leçon: exemples. D'autres portent sur l'écrit, l'oral, la lecture littéraire: Écriture: diversité des situations; Exploration des situations de prise de parole; Lecturellittérature: enseignement continué. L'analyse de dispositifs se fait aussi en lien à des genres d'activité scolaire: Présentation des cercles de lecture; Etude de textes et lecture; La lecture suivie: exemples pour chaque année du CO; Lecture rapide d'extraits de pièces et proposition d'analyse pour une leçon. Enfin, les analyses sont mises en rapport avec les gestes de l'enseignant: Analyse à priori d'une tâche et d'une activité; Problème des consignes; Évaluer un questionnaire. Le second groupe intègre tous les items touchant à la conception et à l'animation des dispositifs didactiques. Nous retrouvons à peu de choses près la même structure que pour les items de l'analyse de dispositifs, à savoir des items généraux sur les séquences: Comment construire une leçon ou une séquence: exemples; Création d'une SD: préparer des modules. Nous avons répertorié également des items qui portent spécifiquement sur des activités liées à l'écrit, à la structuration de la langue ou à la lecture-littéraire: Concevoir en groupe des activités autour d'une nouvelle; Mise en pratique: la lecture. Des items traitent d'un genre d'activités scolaire: Mise en pratique de la dictée ou relèvent des gestes de l'enseignant: Rédiger consigne PI et PF; élaborer un questionnaire; Mise en pratique de la gestion d'une leçon (le timing). 
La sous-catégorie Réguler intègre des items portant sur la différenciation des situations d'enseignement et sur l'évaluation. Cette rubrique comporte 22 des 134 items de la catégorie. Nous n'avons relevé qu'un seul item relatif à la différenciation, Réguler des séquences d'enseignementlapprentissage dans la branche. La majorité des items (21) porte sur l'évaluation. Il peut s'agir d'une réflexion sur l'évaluation de manière générale, L'évaluation; Évaluation diagnostiquelformativel sommative; Relation entre objet d'enseignement et évaluation, Analyse à postériori des évaluations. Il peut être question de l'évaluation d'un domaine particulier du français: Evaluer l'orthographe dans le cadre d'une SD; Évaluation de la compréhension/production de textes à valeur littéraire. D'autres items concernent davantage les pratiques de correction: Formulation des critères de correction; Conception d'une grille d'évaluation pour divers genres de textes; Mise en pratique: grilles de correction et d'évaluation; Répartition des points.

La sous-catégorie S'approprier les outils de l'enseignement présente des items à la fois sur la définition et l'analyse des supports d'enseignement et sur des outils non disciplinaires comme l'usage des technologies de la communication et des médias. Les occurrences sont au nombre de 21 sur 134. Les items en lien aux supports concernent les moyens d'enseignement de façon générique - La séquence-la leçon: les manuels - ou invitent à leur évaluation: La qualité du support (exemples). Aussi, certains items sont spécifiques à une sous-discipline du français: pour la lecture, Présentation de Lector-Lectrix, Aider les lecteurs faibles, pour des questions de structuration de la langue, Enseignement de la grammaire: manuels (Nathan-Hatier) ou encore concernant l'apprentissage de genres textuels particuliers, Enseignement de la poésie: 2. Les manuels. Au sujet des outils non disciplinaires, nous retrouvons majoritairement des items qui sont en liens avec les nouvelles technologies de l'information et de la communication, comme Mitic par exemple, ou encore avec Reconnaitre des situations d'apprentissage qui favorisent l'intégration des connaissances [...] en utilisant les médias.

Créer de la mémoire didactique est un autre geste enseignant mis en évidence dans les descriptifs. Les items qui l'évoquent concernent la Mise en pratique de l'ancrage et la mise en commun ou encore le Rappel sur les objectifs. Cette sous-catégorie regroupe 6 items sur les 134, ce qui correspond à seulement 4,5\% des contenus de la catégorie Enseignant.

Ce sont les contenus de savoir sur la pratique qui comptent le moins d'items, avec 7 unités sur $253(2.8 \%)$. Les contenus de ces items visent la pratique réflexive: Analyse de la pratique enseignante en production de textes, Analyse d'un évènement remarquable, Analyser sa propre pratique, émettre des hypothèses sur les résultats observés, évoquer des démarches alternatives et Analyse réflexive de leçons et de supports d'enseignement.

Plus de la moitié des contenus des descriptifs des institutions de formation à 
l'enseignement secondaire portent sur les situations et les gestes didactiques. L'analyse, la conception et la mise en œuvre de dispositifs sont valorisées. Ce sont les contenus de savoir en lien à la discipline scolaire «français» qui viennent en seconde importance; nous avons relevé la forte présence des contenus en lien à la grammaire, aux théories du langage et des genres textuels, à la littérature et à l'histoire de l'enseignement secondaire. Moins de $12 \%$ des contenus totaux sont consacrés aux catégories «l'élève et son apprentissage», «l'institution» et «la praxéologie». Les contenus phares des items de ces trois catégories sont respectivement l'analyse de textes d'élèves, les plans d'études et l'analyse réflexive.

\section{Mise en parallèle des résultats selon les institutions}

Si nous observons la distribution des contenus des descriptifs pour chacune des institutions, il est possible de constater des tendances fortes et de dégager les similitudes ou différences entre celles-ci. Le tableau 3 montre la répartition et le pourcentage respectif des items par catégories et sous-catégories. La lecture des différents pourcentages doit se faire horizontalement, de manière à mieux comprendre la répartition des items de contenu dans chacune des institutions.

À l'IUFE, nous observons qu'une place importante est donnée aux savoirs sur le français et ses disciplines (40,4\%), à l'intérieur desquels les théories du langage $(7,1 \%)$ et l'histoire de l'enseignement $(6,1 \%)$ prennent le dessus. La deuxième catégorie en importance concerne les savoirs sur les situations et les gestes didactiques (39,4\%). Trois éléments émergent de manière significative: analyser des dispositifs didactiques (22,2\%), concevoir et animer des dispositifs didactiques (6,1\%), définir, s'approprier des outils non disciplinaires: TIC, TN, etc. $(5,1 \%)$. Trois catégories restent cependant minoritaires: stages pratiques $(5,1 \%)$, savoir sur les élèves et leurs apprentissages $(7,1 \%)$ et contenus de savoir sur les prescriptions institutionnelles $(2 \%)$.

Pour la HEP Béjune, nous retrouvons des résultats similaires. Les contenus de savoir sur les français et ses disciplines $(50 \%)$ sont constitués majoritairement par la didactique de l'expression écrite $(14,3 \%)$ et la didactique de la lecture et de la littérature (14,3\%). Quant aux contenus de savoir sur les situations et les gestes didactiques $(28,6 \%)$, ils sont mis en exergue respectivement par l'analyse de dispositifs didactiques (7,1\%), l'évaluation (7,1\%), la définition, la description et l'analyse des supports d'enseignement $(7,1 \%)$ et l'appropriation d'outils non disciplinaires: TIC, TN, etc. (7,1\%). Enfin, les contenus de savoir sur les élèves et leurs apprentissages ne figurent dans les plans d'étude qu'à la hauteur de $7.1 \%$ avec, notamment, l'analyse de textes d'élèves. Deux catégories ne contiennent aucune unité de contenu: il s'agit du savoir sur les prescriptions institutionnelles et des stages pratiques. 
Tableau 3: Répartition des items de contenu du secondaire par catégories et sous-catégories

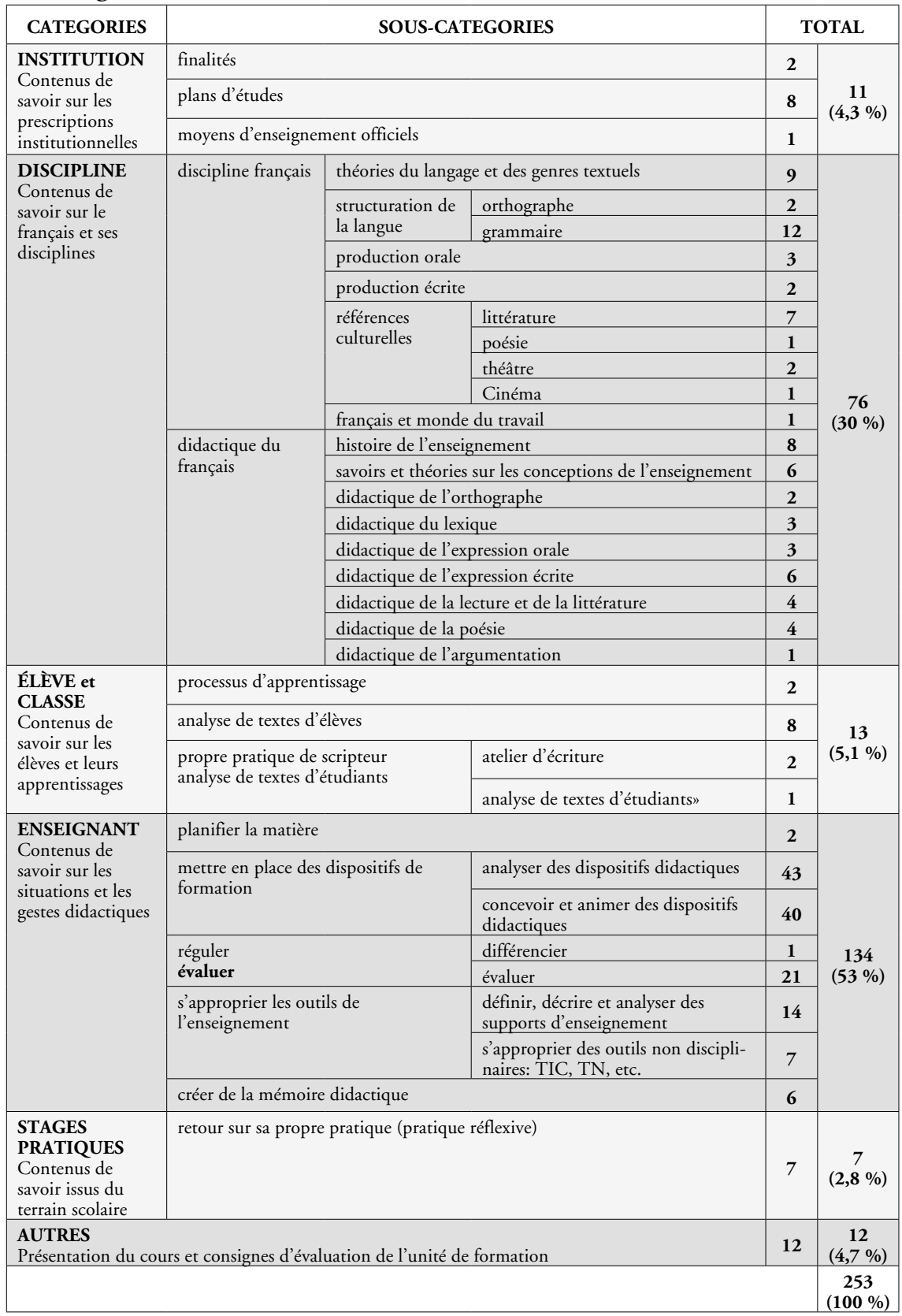


Tableau 4: Poids en pourcentage des catégories et sous-catégories pour chacune des institutions du secondaire

\begin{tabular}{|c|c|c|c|c|c|c|c|c|}
\hline \multirow[b]{2}{*}{ CATÉGORIES } & \multirow{2}{*}{\multicolumn{3}{|c|}{ SOUS-CATEGORIES }} & \multicolumn{5}{|c|}{ INSTITUTIONS } \\
\hline & & & & $\begin{array}{l}\% \\
\text { IUFE }\end{array}$ & $\begin{array}{l}\% \text { HEP } \\
\text { BEJUNE }\end{array}$ & $\begin{array}{l}\% \text { HEP } \\
\text { VALAIS }\end{array}$ & $\begin{array}{l}\% \text { HEP } \\
\text { VAUD }\end{array}$ & $\begin{array}{l}\% \text { UNI } \\
\text { FRI }\end{array}$ \\
\hline \multirow{3}{*}{$\begin{array}{l}\text { INSTITUTION } \\
\text { Contenus de } \\
\text { savoir sur les } \\
\text { prescriptions } \\
\text { institutionnelles }\end{array}$} & \multicolumn{3}{|l|}{ finalités } & - & - & - & $8 \%$ & - \\
\hline & \multicolumn{3}{|c|}{ plans d'études } & $2 \%$ & - & - & $8 \%$ & $4,2 \%$ \\
\hline & \multicolumn{3}{|c|}{ moyens d'enseignement officiels } & - & - & - & - & $1,1 \%$ \\
\hline & & & & $2 \%$ & $0 \%$ & $0 \%$ & $16 \%$ & $5,3 \%$ \\
\hline \multirow{19}{*}{$\begin{array}{l}\text { DISCIPLINE } \\
\text { Contenus de } \\
\text { savoir sur le } \\
\text { français et ses } \\
\text { disciplines }\end{array}$} & \multirow{10}{*}{$\begin{array}{l}\text { contenus de } \\
\text { savoir sur la } \\
\text { discipline } \\
\text { français }\end{array}$} & \multicolumn{2}{|c|}{$\begin{array}{l}\text { théories du langage et des } \\
\text { genres textuels }\end{array}$} & $7,1 \%$ & - & $5 \%$ & - & - \\
\hline & & \multirow{2}{*}{$\begin{array}{l}\text { structuration } \\
\text { de la langue }\end{array}$} & orthographe & $1 \%$ & - & $5 \%$ & - & - \\
\hline & & & grammaire & $4 \%$ & $7,1 \%$ & - & - & $7,4 \%$ \\
\hline & & \multicolumn{2}{|c|}{ production orale } & $3 \%$ & - & - & - & - \\
\hline & & \multicolumn{2}{|c|}{ production écrite } & $1 \%$ & - & - & - & $1,1 \%$ \\
\hline & & \multirow{4}{*}{$\begin{array}{l}\text { références } \\
\text { culturelles }\end{array}$} & littérature & $2 \%$ & - & - & - & $5,3 \%$ \\
\hline & & & poésie & - & - & - & - & $1,1 \%$ \\
\hline & & & théâtre & $1 \%$ & - & - & - & $1,1 \%$ \\
\hline & & & Cinéma & - & - & - & - & $1,1 \%$ \\
\hline & & \multicolumn{2}{|c|}{$\begin{array}{l}\text { français et monde du } \\
\text { travail }\end{array}$} & - & - & - & - & $1,1 \%$ \\
\hline & \multirow{9}{*}{$\begin{array}{l}\text { contenus de } \\
\text { savoir sur la } \\
\text { didactique } \\
\text { du français }\end{array}$} & \multicolumn{2}{|c|}{ histoire de l'enseignement } & $6,1 \%$ & - & - & $4 \%$ & $1,1 \%$ \\
\hline & & \multicolumn{2}{|c|}{$\begin{array}{l}\text { savoirs et théories sur } \\
\text { l'enseignement }\end{array}$} & - & - & $5 \%$ & $12 \%$ & $2,1 \%$ \\
\hline & & \multicolumn{2}{|c|}{$\begin{array}{l}\text { didactique de l'ortho- } \\
\text { graphe }\end{array}$} & $1 \%$ & $7,1 \%$ & - & - & - \\
\hline & & \multicolumn{2}{|c|}{ didactique du lexique } & $2 \%$ & $7,1 \%$ & - & - & - \\
\hline & & \multicolumn{2}{|c|}{$\begin{array}{l}\text { didactique de l'expression } \\
\text { orale }\end{array}$} & $2 \%$ & - & - & $4 \%$ & - \\
\hline & & \multicolumn{2}{|c|}{$\begin{array}{l}\text { didactique de l'expression } \\
\text { écrite }\end{array}$} & $4 \%$ & $14,3 \%$ & - & - & - \\
\hline & & \multicolumn{2}{|c|}{$\begin{array}{l}\text { didactique de la lecture et } \\
\text { de la littérature }\end{array}$} & $2 \%$ & $14,3 \%$ & - & - & - \\
\hline & & \multicolumn{2}{|c|}{ didactique de la poésie } & $4 \%$ & - & - & - & - \\
\hline & & \multicolumn{2}{|c|}{$\begin{array}{l}\text { didactique de l'argumen- } \\
\text { tation }\end{array}$} & $0 \%$ & $0 \%$ & - & - & $1,1 \%$ \\
\hline & & & & $40,4 \%$ & $50 \%$ & $15 \%$ & $20 \%$ & $22,1 \%$ \\
\hline
\end{tabular}

\begin{tabular}{|c|c|c|c|c|c|c|c|}
\hline \multirow{4}{*}{$\begin{array}{l}\text { ÉLÈVE et } \\
\text { CLASSE } \\
\text { Contenus de } \\
\text { savoir sur les } \\
\text { élèves et leurs } \\
\text { apprentissages }\end{array}$} & \multicolumn{2}{|c|}{ processus d'apprentissage } & - & - & - & $8 \%$ & - \\
\hline & \multicolumn{2}{|c|}{ analyse de textes d'élèves } & $4 \%$ & $7,1 \%$ & $5 \%$ & $4 \%$ & $1,1 \%$ \\
\hline & \multirow{2}{*}{$\begin{array}{l}\text { contenus de } \\
\text { savoir sur } \\
\text { sa propre } \\
\text { pratique de } \\
\text { scripteur }\end{array}$} & atelier d'écriture & $2 \%$ & - & - & - & - \\
\hline & & $\begin{array}{l}\text { analyse de textes } \\
\text { d'étudiants }\end{array}$ & $1 \%$ & - & - & - & - \\
\hline & & & $7,1 \%$ & $7,1 \%$ & $5 \%$ & $12 \%$ & $1,1 \%$ \\
\hline
\end{tabular}




\begin{tabular}{|c|c|c|c|c|c|c|c|}
\hline \multirow{8}{*}{$\begin{array}{l}\text { ENSEIGNANT } \\
\text { Contenus de } \\
\text { savoir sur les } \\
\text { situations et les } \\
\text { gestes didac- } \\
\text { tiques }\end{array}$} & \multicolumn{2}{|c|}{ planifier la matière } & $1 \%$ & - & $5 \%$ & - & - \\
\hline & \multirow{2}{*}{$\begin{array}{l}\text { mettre } \\
\text { en place } \\
\text { des dispo- } \\
\text { sitifs de } \\
\text { formation }\end{array}$} & $\begin{array}{l}\text { analyser des dispositifs } \\
\text { didactiques }\end{array}$ & $22,2 \%$ & $7,1 \%$ & $10 \%$ & $20 \%$ & $13,7 \%$ \\
\hline & & $\begin{array}{l}\text { concevoir et animer des } \\
\text { dispositifs didactiques }\end{array}$ & $6,1 \%$ & - & $5 \%$ & $12 \%$ & $31,6 \%$ \\
\hline & \multirow[t]{2}{*}{ réguler } & différencier & - & - & $5 \%$ & - & - \\
\hline & & évaluer & $1 \%$ & $7,1 \%$ & $55 \%$ & $4 \%$ & $7,4 \%$ \\
\hline & \multirow{2}{*}{$\begin{array}{l}\text { s'approprier } \\
\text { les outils } \\
\text { de l'ensei- } \\
\text { gnement }\end{array}$} & $\begin{array}{l}\text { définir, décrire et analyser } \\
\text { des supports d'ensei- } \\
\text { gnement }\end{array}$ & $4 \%$ & $7,1 \%$ & - & $4 \%$ & $8,4 \%$ \\
\hline & & $\begin{array}{l}\text { s'approprier des outils } \\
\text { non disciplinaires: TIC, } \\
\text { TN, etc, }\end{array}$ & $5,1 \%$ & $7,1 \%$ & - & $4 \%$ & - \\
\hline & \multicolumn{2}{|c|}{ créer de la mémoire didactique } & - & - & - & - & $6,3 \%$ \\
\hline & & & $39,4 \%$ & $28,6 \%$ & $80 \%$ & $44 \%$ & $67,4 \%$ \\
\hline
\end{tabular}

\begin{tabular}{|l|l|c|c|c|c|c|}
\hline $\begin{array}{l}\text { STAGES } \\
\text { PRATIQUES } \\
\begin{array}{l}\text { Savoirs praxéolo- } \\
\text { giques }\end{array}\end{array}$ & $\begin{array}{l}\text { retour sur sa propre pratique (pratique } \\
\text { réflexive) }\end{array}$ & $5,1 \%$ & - & - & $8 \%$ & - \\
\hline
\end{tabular}

\section{AUTRES}

Présentation du cours et consignes d'évaluations de l'unité de formation

\begin{tabular}{|c|c|c|c|c|}
\hline $6,1 \%$ & $14,3 \%$ & - & - & $4,2 \%$ \\
\hline $6,1 \%$ & $14,3 \%$ & $0 \%$ & $0 \%$ & $4,2 \%$ \\
\hline$\underline{100} \%$ & $\underline{100} \%$ & $\underline{100} \%$ & $\underline{100} \%$ & $\underline{100} \%$ \\
\hline
\end{tabular}

Pour ce qui est de la HEP Valais, deux catégories se retrouvent bien présentes, mais dans des proportions différentes: les contenus de savoir sur les situations et les gestes didactiques sont dominants et rassemblent $80 \%$ des items de contenu, avec notamment les composantes évaluer (55\%) et analyser des dispositifs $(22.2 \%)$. Pour ce qui est des contenus de savoir sur le français et ses disciplines (15\% de la totalité des items), ils sont constitués par les théories $d u$ langage (5\%), l'orthographe (5\%) et les savoirs et les théories sur l'enseignement $(5 \%)$. Les contenus de savoir sur les élèves et leurs apprentissages comptabilisent $5 \%$ des items uniquement constitués par l'analyse de textes d'élèves. Les catégories Savoir sur les prescriptions institutionnelles et Stages pratiques, quant à elles, équivalent à $0 \%$.

A la HEP Vaud, l'importance donnée aux catégories de contenus de savoir sur les enseignants et sur la discipline est forte: les contenus de savoir sur les situations et les gestes didactiques concernent $44 \%$ des items avec notamment analyser des dispositifs didactiques $(20 \%)$ et concevoir et animer des dispositifs didactiques (12\%), et les contenus de savoir sur le français et ses disciplines touchent près de $20 \%$ avec les savoirs et théories sur l'enseignement (12\%). Reste que pour cette institution, les contenus de savoir sur les prescriptions insti- 
tutionnelles sont également bien marqués (16\%) avec les finalités (8\%) et les plans d'études $(8 \%)$. Toutes les autres catégories sont présentes et se partagent avec plus ou moins de force les autres unités de contenu: contenus de savoir sur les élèves et leurs apprentissages ( $12 \%)$ et Stages pratiques ( $8 \%)$,

Enfin, l'on retrouve à l'Université de Fribourg une prépondérance des contenus de savoir sur les situations et les gestes didactiques $(67,4 \%)$, lesquels traitent surtout de la conception et l'animation de dispositifs didactiques $(31,6 \%)$ et de l'analyse de dispositifs didactiques (13,7\%). Quant aux contenus de savoir sur le français et ses disciplines, représentés dans 22,1\% des items, ils traitent de la grammaire et des références culturelles sur la littérature. Deux autres catégories de contenus de savoir sont moins marquées: les contenus de savoir sur les prescriptions institutionnelles et les contenus de savoir sur les élèves et leurs apprentissages indiquent des pourcentages faibles, 5,3\% et $1,1 \%$. Quant aux contenus de savoir pour les stages pratiques, ils ne sont pas représentés

Nous remarquons une constante dans l'ensemble des institutions de formation: les contenus de savoir traitant de la discipline français et ceux liés aux dispositifs et gestes de l'enseignant prennent le dessus. Ces contenus de savoir sont majoritaires, mais dans des proportions différentes: les items de contenu de l'IUFE et de la HEP Bejune sont davantage centrés sur les contenus de savoir liés à la discipline, tandis que ceux de la HEP Valais, la HEP Vaud et l'Université de Fribourg portent sur les situations et les gestes didactiques. Les contenus de savoir disciplinaires mis en exergue concernent prioritairement la grammaire, l'histoire de l'enseignement et les contenus de savoir et théories sur l'enseignement. Les contenus de savoir en lien aux dispositifs et aux gestes abordent l'analyse, la conception et l'animation de dispositifs didactiques, en réservant une place privilégiée à l'évaluation. Quant aux contenus de savoir sur les élèves et leurs apprentissages, présents dans tous les descriptifs, mais de manière moins forte, ils rassemblent essentiellement des activités centrées sur l'analyse de textes d'élèves (Gagnon, Surian, Mosquera Roa \& Dolz, à paraitre)

\section{Conclusion}

La reconstitution des différents cursus de formation à l'enseignement secondaire montre une grande variété, témoignant d'une volonté de rendre la formation accessible et adaptable aux besoins du marché professionnel. Cependant, les intitulés des unités de formation et leur organisation à l'intérieur du cursus montrent peu de précision quant à l'objet didactique du français et au traitement de ses sous-disciplines. Ce sont les aspects liés à l'analyse de pratiques et aux gestes professionnels qui sont davantage mis en lumière.

La répartition des items de contenu des descriptifs montre la prédominance des contenus d'ingénierie didactique. Il importe d'amener le formé à analyser, 
concevoir et mettre en oeuvre des dispositifs d'enseignement. Ces contenus sont abordés dans les descriptifs de toutes les institutions de formation à l'enseignement secondaire romandes. Cette catégorie de contenus se voit concurrencée par une autre, celle des contenus de savoir disciplinaires. Ces derniers portent sur la grammaire, les théories du langage et des genres textuels, la littérature et l'histoire de l'enseignement. Le poids des pratiques antérieures est ici difficile à cerner. Par exemple, il n'y a pas d'uniformité entre les contenus des HEP et ceux des deux institutions universitaires. Nous pensions observer davantage de similitudes entre une institution jeune comme la HEP Valais et ses institutions de filiation, la HEP Vaud et l'université de Fribourg.

Une entrée par un objet précis, l'enseignement de la production écrite, permet de délimiter un tant soit peu les contenus de savoir de la formation. Notre analyse des descriptifs révèle un objet "production écrite» dillué dans le champ plus vaste de la didactique du français. Certaines sous-catégories émergent toutefois: expression et production écrite, analyse de textes d'élèves, pratique du scripteur.

La présente analyse, nous l'avons dit, consiste en une première étape en vue d'un examen des pratiques effectives de formation. Certaines de nos interrogations ne trouvent réponse, notamment celles qui concernent l'organisation de l'alternance et la place concrète des contenus de savoir de la pratique dans la présentation des contenus de formation dans les structures. Toutefois, nous sommes en mesure de formuler des hypothèses quant aux zones de divergence ou de convergence entre les institutions. Cette analyse des documents officiels et des descriptifs permet de proposer un premier découpage des contenus susceptibles d'être abordés dans les pratiques. D'ailleurs, la rédaction du descriptif exige du formateur d'établir un ordre dans les contenus, de mettre à plat ce qu'il a l'intention de traiter. Ainsi, nous tâchons de relever un défi de taille: structurer, hiérarchiser les contenus impliqués dans le processus étagé et complexe qu'est la formation à l'enseignement de la production écrite.

\section{Notes}

1 Le projet s'intitule Objets et processus de la formation en didactique du français. Analyse de l'activité des formateurs d'enseignants du primaire et du secondaire en production écrite (Fonds National suisse de la recherche scientifique, numéro 100014_126682). Il est sous la direction de Joaquim Dolz.

2 Une unité de formation correspond à un enseignement suivi à l'intérieur d'une plage horaire déterminée. L'intitulé, générique, regroupe des modalités d'exposition différentes des contenus: atelier, cours magistral, conférence, séminaire de recherche, etc.

3 Notons encore que pour les cantons romands seul Fribourg proposait deux filières distinctes.

4 Conférence suisse des directeurs cantonaux de l'instruction publique.

5 Conférence intercantonale de l'instruction publique de la Suisse romande et du Tessin.

6 Nous ne détaillerons pas les items de contenu de cette catégorie dans la suite du texte étant donné qu'elle ne donne rien à voir sur les contenus de savoir en jeu dans la formation des enseignants. 


\section{Références bibliographiques}

Chervel, A. (1998). L'histoire des disciplines scolaires. In A. Chervel, La culture scolaire. Une approche historique (pp. 9-57). Paris: Belin.

Chevallard, Y. (1999). L'analyse des pratiques enseignantes en théorie anthropologique du didactique. Recherches en Didactique des Mathématiques, 19(2), 221-266.

Criblez, L. (2004). La formation des enseignants du secondaire en Suisse. In Résonances, 4. Page consultée le 7 décembre 2012 au http://www.ordp.vsnet.ch/fr/resonance/2004/d\%E9 cembre/criblez.htm.

Desjardins, J. \& Dezutter, O. (2009). Développer des compétences professionnelles en formation initiale à l'enseignement préscolaire et primaire: regard sur l'organisation des programmes en contexte québécois. Canadian Journal of Education, 32, (4), 873-902.

Dolz, J, Schneuwly, B. \& Thévenaz-Christen, Th. (2008). L'articulation vygotskienne entre objet enseigné et outil médiateur comme fondement de la didactique. In M. Brossard \& J. Fijalkow (Éd.), Vygotski et les recherches en éducation et en didactiques (pp. 143-156). Bordeaux: Presses Universitaires de Bordeaux.

Extermann, B. (2007). La professionnalisation émergente des enseignants secondaires dans un champ de tensions: étude historique d'un moment clé de l'évolution de la formation pédagogique à Genève (1975-1977). Actes du Colloque «Qu'est-ce qu'une formation professionnelle universitaire des enseignants?», IUFM Nord-Pas de Calais.

Page consultée le 5 novembre 2015 au http://www.lille.iufm.fr/IMG/pdf/109-117_ EXTERMANN_Tome1.pdf.

Gagnon, R. \& Surian, M. (2012). Quels savoirs pour l'enseignement du français dans les plans de formation suisses romands? Repères, 44, 93-115.

Gagnon, R., Surian, M., Mosquera Roa., S. \& Dolz, J. (à paraitre). Textes d'élèves et d'étudiants: supports et outils de la formation en didactique du français. Français aujourd'hui, 184.

Gaillard, X. (2004). Devenir enseignant-e dans le secondaire I ou II... en Valais. In Résonances, 4. Page consultée le 21 janvier 2013 au http://www.ordp.vsnet.ch/fr/resonance/2004/d\%E9cembre/sommaire.htm.

Goigoux, R., Ria, L. \& Toczek-Capelle, M.-C. (2009). Mieux connaître les parcours de formation des enseignants débutants pour mieux les former. In R. Goigoux, L. Ria et M.C. Toczek-Capelle, (Éds.) Les parcours de formation des enseignants débutants (pp. 25-44). Clermont: Presses Universitaires Blaise-Pascal.

Hofstetter, R. \& Schneuwly, B. (2009). Savoirs en (trans)formation. Au cœur des professions de l'enseignement et de la formation. In R. Hofstetter \& B. Schneuwly, Savoirs en (trans) formations des savoirs dans les formations aux professions enseignantes (pp. 6-22). Bruxelles: De Boeck.

Lenoir, Y. \& Vanhulle, S. (2006). Étudier la pratique enseignante dans sa complexité. Une exigence pour la recherche et la formation à l'enseignement. In A. Hasni, Y. Lenoir \& J. Lebeaume (Éds.), La formation à l'enseignement des sciences et des technologies au secondaire dans le contexte des réformes par compétences (pp. 193-245). Québec: Presses de l'Université du Québec.

Lussi Borer, V. (2009). Les savoirs: un enjeu crucial de l'institutionnalisation des formations à l'enseignement. In R. Hofstetter \& B. Schneuwly, Savoirs en (trans)formations des savoirs dans les formations aux professions enseignantes (pp. 41-58). Bruxelles: De Boeck.

Noverraz, D. (2008). La formation des maitres dans le canton de Vaud, du XVIe siècle au XXI siècle. Yens sur Morges: Éditions Cabédita.

Schneuwly, B. \& Dolz, J. (avec Aeby-Daghé, S., Bain, D., Canelas-Trevisi, S., Sales Cordeiro, G., Gagnon, R., Jacquin, M., Ronveaux, Ch., Thévenaz-Christen, Th. \& Toulou, S.) (2009). Des objets enseignés en classe de français; le travail de l'enseignant sur la rédaction de textes argumentatifs et sur la subordonnée relative. Rennes: Presses universitaires de Rennes. 
Mots-clés: Institution de formation secondaire, didactique du français, enseignement de la production écrite, plan d'études, descriptif, unité de formation, item de contenu

\section{Didaktik Französisch: Lehrplangerechter Unterricht der schriftlichen Produktion auf der Sekundarstufe I in der Romandie}

\section{Zusammenfassung}

Unser Beitrag ist Teil eines umfassenderen Forschungsprojekts über die Ausbildungspraxis für das Unterrichten von schriftlicher Produktion. Zwecks eines besseren Verständnisses der effektiven Ausbildungspraxis ist im Vorfeld eine Untersuchung der institutionellen Rahmenbedingungen des Sekundarstufen-I-Unterrichts unerlässlich. Deshalb befassen wir uns zunächst mit den institutionellen Faktoren und Bedingungen, die dem Sekundarstufen-I-Unterricht in der Romandie (französischsprachigen Schweiz) zugrunde liegen. Anschliessend geht es uns um die Gewichtung der Ausbildungseinheiten der Fachdidaktik Französisch - Kurse und Seminare - innerhalb des gesamten Angebots an Lehrveranstaltungen für das Lehramt der Sekundarstufe I. Anhand einer sorgfältigen Analyse der Inhaltsbeschreibungen der Ausbildungseinheiten unternehmen wir den Versuch einer Kategorisierung und unterscheiden fünf Gruppen von Wissensinhalten in Bezug auf die Institution, das Fach, die Schüler und die Klasse, die Lehrtätigkeit sowie die Unterrichtspraxis. So dokumentieren wir die Gemeinsamkeiten und Unterschiede zwischen den verschiedenen mit der Ausbildung von Lehrkräften der Sekundarstufe I befassten Institutionen der Romandie und zeigen die Tendenzen und Prioritäten bei der Wissensvermittlung am Beispiel der schriftlichen Produktion auf.

Schlagworte: Ausbildungsinstitutionen, Lehramt der Sekundarstufe I, Fachdidaktik Französisch, Unterricht der schriftlichen Produktion, Lehrplan, Beschreibung, Lehreinheit, Inhalte 


\section{Quali saperi per l'insegnamento del francese e della produzione scritta alla scuola secondaria nei piani della formazione svizzera romanda?}

\section{Riassunto}

Il nostro contributo si inscrive in un vasto progetto di ricerca sulle pratiche di formazione all'insegnamento della produzione scritta. Un'analisi a monte delle prescrizioni istituzionali delle istituzioni all'insegnamento secondario è inevitabile per capire le pratiche effettive dei formatori. L'oggetto di questo articolo mira a mettere in luce, in un primo tempo, i fattori e i limiti istituzionali all'insegnamento secondario in Svizzera romanda che agiscono sulle istituzioni. In un secondo momento, situiamo le unità di formazione - corsi e seminari - dedicate alla didattica del francese nell'insieme dei corsi proposti per la formazione degli insegnanti della scuola secondaria. Infine, attraverso un'analisi dettagliata dei contenuti dei descrittivi delle unità di formazione, effettuiamo una prova di categorizzazione di contenuti del sapere. Quest'ultima individua cinque insiemi di contenuti di sapere: istituzionali, disciplinari, legati all'allievo e alla classe, legati al lavoro dell'insegnante e alla pratica. Attraverso questo lavoro, evidenziamo le similitudini e le divergenze tra le diverse istituzioni di formazione all'insegnamento secondario in maniera esaustiva. Tracciamo, così, le tendenze che permettono di cogliere le priorità per l'insegnamento della produzione scritta nelle istituzioni di formazione svizzero-romande nell'ambito dell'insegnamento della produzione scritta al livello secondario.

Parole chiave: Istituzione di formazione secondaria, didattica del francese, insegnamento della produzione scritta, piano di studi, unità di formazione, strumenti di contenuto 


\title{
Which Content of Knowledge for Teaching Written Production in Secondary Education? An Analysis of Secondary Teachers Training Curricula in French Areas of Switzerland
}

\begin{abstract}
Summary
Part of a broader research project on Teacher Education practices for French written production in French areas of Switzerland, this contribution presents a content analysis of course syllabi and programs in secondary education. In order to understand the context of the five institutions responsible for secondary teacher education, we highlight some of the key institutional constraints, as well as results from the history of practice or political decisions. We also reconstruct the organization of the French didactic curriculum in the institutions, questioning the content and elements relating to written production. The last portion of our work is based on a contents analysis of the course syllabi collected in all five institutions. This contents analysis leads us to propose a content knowledge taxonomy for teaching written production in secondary education.
\end{abstract}

Key words: Swiss teacher education institutions, French didactic, teacher education practices, French written production, syllabi, programs, content knowledge taxonomy 